weeks, those requiring tube feeding for more than 14 days being handicapped at follow up.

\title{
SURGERY FOR NEONATAL-ONSET SEIZURES
}

Four children with intractable neonatal-onset seizures treated successfully by hemispherectomy at 1 1/2-5 years of age are reported from UCLA School of Medicine, Los Angeles, California. Positron emission tomography (PET) with fluoro-D-glucose provided accurate localization of seizure foci whereas CT and MRI were either normal or showed mild generalized cerebral atrophy. The report illustrates the important role of PET in the evaluation of children with intractable epilepsy of neonatal onset. (Chugani HT et al. Surgical treatment of intractable neonatal-onset seizures: The role of positron

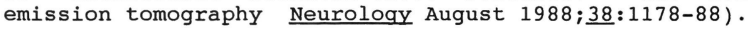

COMMENT. The criteria for hemispherectomy were as follows: 1. Intractable unilateral seizures with diffuse epileptic activity in the affected hemisphere. 2. Persistent neurologic deficit on the contralateral side. 3. Malfunction of the affected hemisphere and intact function of the opposite hemisphere as tested by interictal EEGs, evoked potentials, thiopental test, and PET. At UCLA the results of surgery are impressive: the patients were seizure-free for periods up to 1 $1 / 2$ years and 3 patients were off all anticonvulsants. The surgical approach to treatment of refractory seizures appears superior to the conservative method with potentially toxic anticonvulsant drugs. The authors are to be complemented for their aggressive approach and search for alternate forms of early treatment.

\section{NEONATAL CEREBRAL HEMORRHAGE AND ISCHEMIC LESIONS}

\section{SHUNTS FOR POST-HEMORRHAGIC HYDROCEPHALUS}

The outcome of 19 infants who underwent cerebrospinal shunting for post-hemorrhagic ventricular dilatation is reported from the Department of Pediatrics and Neonatal Medicine, Hammersmith Hospital, London W12. Periventricular hemorrhages were diagnosed by ultrasound scanning, and surgery was considered necessary if the hydrocephalus could not be controlled by intermittent lumbar or ventricular tapping and the CSF pressure was above $6 \mathrm{mmHg}$. Complications of ventriculo-peritoneal shunts included seizures at the time of the surgery in 8 infants, postoperative infection in 12 of 58 (20\%) procedures and blockage of 29 shunts. Shunt infection with Staphylococcal epidermidis occurred in almost half the patients in spite of prophylactic antibiotics. Shunt blockage occurring in $70 \%$ of infants was less frequent in those over $2.5 \mathrm{~kg}$ and with CSF protein below 1g/1. Long-term outcome was poor: 3 died, 4 were quadriplegic and mentally retarded, and only 4 (20\%) were developmentally normal. Outcome was correlated with pre-operative parenchymal brain lesions diagnosed by ultrasound scans. (Hislop JE et al. Outcome of infants 